\title{
A technique to improve optical time division multiplexing - wavelength division multiplexing performance
}

\author{
C. C. Hiew ${ }^{1}$, F. M. Abbou ${ }^{2 a)}$, T. H. Chuah ${ }^{1}$, \\ and Hairul A. Abdul-Rashid ${ }^{1}$ \\ ${ }^{1}$ Faculty of Engineering, \\ Multimedia University, 63100 Cyberjaya, Malaysia \\ ${ }^{2}$ Alcatel Network System, \\ 50450 Kuala Lumpur, Malaysia \\ a)fouad@mти.edu.my
}

\begin{abstract}
Optical time division multiplexing - wavelength division multiplexing (OTDM-WDM), intensity modulation (IM) systems employing optical amplification and dispersion management (DM) are analyzed with and without the introduction of an optical narrowband bandpass filter placed right after optical time demultiplexing. The use of the filter was found to improve the performance of the demultiplexed channels by reducing amplitude jitter while possessing high intersymbol interference (ISI) tolerance. The OTDM-WDM link shows good performance up to $2000 \mathrm{~km}$ with the OTDM filter providing a Q gain of 0.7 $-1.1 \mathrm{~dB}$. The optimal input power reduces for longer distances due to increased significance of nonlinear effects. A $900 \mathrm{~km}$ link showed good performance for spectral efficiencies as low as $0.52 \mathrm{bits} / \mathrm{s} / \mathrm{Hz}$, though the $\mathrm{Q}$ gain provided by the OTDM filter reduces as channel spacing decreases.
\end{abstract}

Keywords: Fiber optic communication, wavelength division multiplexing, optical time division multiplexing, nonlinear effects, dispersion management, bit error rate analysis

Classification: Photonics devices, circuits, and systems

\section{References}

[1] M. Nakazawa, "Solitons for Breaking Barriers to Terabit/Second WDM and OTDM Transmission in the Next Millenium," IEEE J. Select. Topics Quantum Electron., vol. 6, pp. 1332-1343, Nov./Dec. 2000

[2] J. X. Cai, M. Nissov, C. R. Davidson, A. N. Pilipetskill, G. Mohs, H. Li, Y. Cai, E. A. Golovchenko, A. J. Lucero, D. G. Foursa, and N. S. Bergano, "Long-Haul 40 Gb/s DWDM Transmission With Aggregate Capacities Exceeding $1 \mathrm{~Tb} / \mathrm{s}, "$ J. Lightwave Technol., vol. 20, pp. 2247-2257, Dec. 2002. 
[3] C. M. Weinert, R. Ludwig, W. Pieper, H. G Weber, D. Breuer, K. Petermann, and F. Kuppers, " $40 \mathrm{~Gb} / \mathrm{s}$ and 4 x $40 \mathrm{~Gb} / \mathrm{s}$ TDM/WDM Standard Fiber Transmission," J. Lightwave Technol., vol. 17, pp. 2276-2284, Nov. 1999.

[4] T. Yu, W. M. Reimer, V. S. Grigoryan, and C.R. Menyuk, "A Mean Field Approach for Simulating Wavelength-Division Multiplexed Systems," IEEE Photon. Technol. Lett., vol. 12, pp. 443-445, April 2000.

[5] P. A. Humblet and M. Azizoglu., "On the bit error rate of lightwave systems with optical amplifiers," J. Lightwave Technol., vol. 9, pp. 15761582, Nov. 1991.

[6] N. S. Bergano, F. W. Kerfoot, and C. R. Davidson, "Margin measurements in optical amplifier systems," IEEE Photon. Technol. Lett., vol. 5, pp. 304306, March 1993.

[7] C. J. Anderson and J. A. Lyle, "Technique for evaluating system performance using $\mathrm{Q}$ in numerical simulations exhibiting intersymbol interference," Electron. Lett., vol. 30, pp. 71-72, Jan. 1994.

\section{Introduction}

The optical fiber offers a vast amount of bandwidth that can be utilized for communication. One of the chief ways of utilizing this is signal multiplexing. Due to the large bandwidth and the associated high bit rates, the multiplexing process is beyond the capabilities of pure electronic methods and has to be implemented optically as well. One important technique is optical time division multiplexing (OTDM), which has demonstrated impressive bit rates [1]. Lately though, the most commonly used multiplexing technique is wavelength division multiplexing (WDM), which can fully utilize the available fiber bandwidth using $10 \mathrm{~Gb} / \mathrm{s}$ or $40 \mathrm{~Gb} / \mathrm{s}$ channels at high spectral efficiency [2]. Recently, the push has been to increase the bit rate per WDM channel and the combination of OTDM and WDM or in short OTDM-WDM has been touted as a potential solution [3]. It is attractive from two opposing perspectives. Firstly, the integration of WDM in OTDM systems is required to maximize the fiber bandwidth utilization, as a pure OTDM system cannot achieve sufficiently high bit rates to do so. Secondly, the integration of OTDM in WDM systems can increase the bit rate per WDM channel to reduce the number of WDM channels required along with the components required to support a channel, resulting in reduced costs.

In this paper, we look into the use of an optical narrowband bandpass filter placed right after OTDM demultiplexing, called the OTDM filter to improve the performance of the OTDM-WDM system. Note that the systems studied are long distance, intensity modulated (IM) systems employing periodic optical amplification and dispersion management (DM). Generally, the systems consist of $N$ OTDM channels of $10 \mathrm{~Gb} / \mathrm{s}$ bit rates multiplexed to form an $(N \times 10) \mathrm{Gb} / \mathrm{s}$ OTDM signal. Each of these OTDM signals are separate WDM channels that are multiplexed as required. The resultant WDM signal is propagated through the optical system and demultiplexed at the receiver. BER or Q-factor analysis is performed on the demultiplexed 
$10 \mathrm{~Gb} / \mathrm{s}$ signals after detection and filtering.

\section{Simulation Details}

The system block diagram is shown in Fig. 1. Each transmitted channel employs return-to-zero (RZ) modulation, which consists of Gaussian pulses with full width half maximum (FWHM) width of 8 ps. The OTDM channel bit rate is $10 \mathrm{~Gb} / \mathrm{s}$, which are multiplexed to form $40 \mathrm{~Gb} / \mathrm{s}$ WDM channels. Both the OTDM and WDM multiplexing processes are assumed ideal. We simulate five adjacent WDM channels with equal channel spacing of $\Delta f \mathrm{GHz}$ and results are obtained from the middle channel (centered at $1550 \mathrm{~nm}$ ). This is sufficient as almost all effects on a WDM channel are caused by its first two spectrally adjacent neighbors [4]. Each OTDM channel is encoded using different pseudo-random bit sequences (PRBS) of length $2^{9}-1$ bits so that they are uncorrelated.

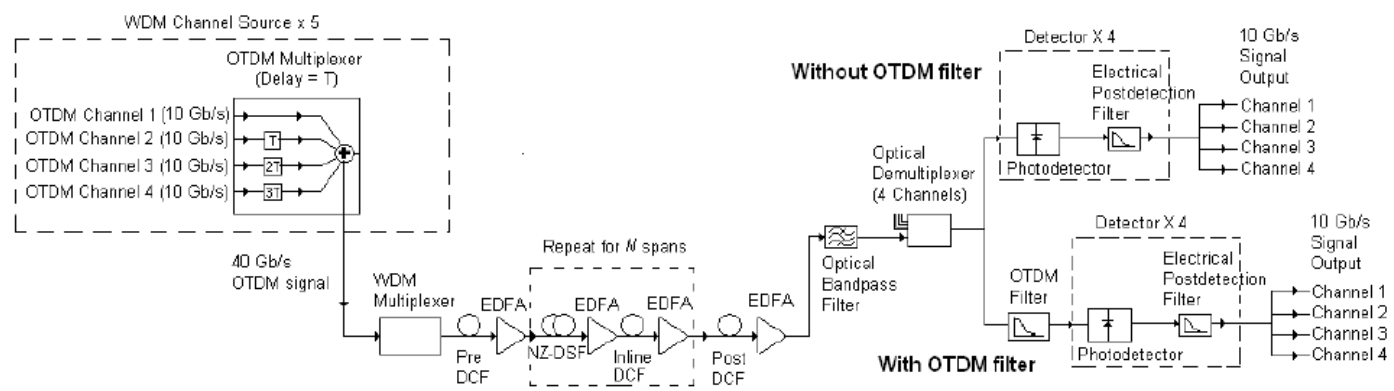

Fig. 1. System block diagram (with or without OTDM filter)

The signal is transmitted across 18 spans of nonzero dispersion shifted fiber (NZ-DSF) of length $50 \mathrm{~km}$ each with dispersion $D=4 \mathrm{ps} / \mathrm{nm} / \mathrm{km}$, dispersion slope $S l=0.1 \mathrm{ps} / \mathrm{nm}^{2} / \mathrm{km}$, effective area $A_{\text {eff }}=55 \mu \mathrm{m}^{2}$ and attenuation $\alpha=0.2 \mathrm{~dB} / \mathrm{km}$. The dispersion compensating fiber (DCF) has $D=-100 \mathrm{ps} / \mathrm{nm} / \mathrm{km}, S l=-2.5 \mathrm{ps} / \mathrm{nm}^{2} / \mathrm{km}, A_{\text {eff }}=30 \mu \mathrm{m}^{2}$ and $\alpha=$ $0.55 \mathrm{~dB} / \mathrm{km}$. The DCF is either used as periodic (inline) dispersion compensation placed after each NZ-DSF span (specified in terms of length, $L_{i n l}$ ) or as pre-dispersion compensation (length, $L_{\text {pre }}$ ) and post-dispersion compensation (length, $L_{\text {post }}$ ). The dispersion management scheme is ideal so that second and third order dispersion cancels out for all channels at the receiver. A second order, super-Gaussian optical bandpass filter with bandwidth $B W_{w d m}$, is used for WDM demultiplexing as this is the shape of many common demultiplexers. OTDM demultiplexing is performed using an ideally square switching window with $92 \%$ duty cycle. All receivers are assumed ideal with no shot and electrical thermal noise considering that the dominant noise in long-range systems is accumulated amplified spontaneous emission (ASE). A five-pole, Bessel postdetection lowpass electric filter is used with single sided bandwidth $B W_{\text {ele }}$. Erbium doped fiber amplifiers (EDFA), with a population inversion factor $n_{s p}$ of 2 , are placed after each fiber span. The 
ASE is modeled as circular complex additive white Gaussian noise (AWGN) with uniform random phase distribution and added onto the WDM signal after each amplifier along the transmission link. The signal and noise are propagated together by solving the nonlinear Schrödinger equation (NLSE) using the split-step Fourier method (SSFM) [4]. Single polarization is assumed for all signals and noise. Multiple runs are performed until the results converge.

To improve the performance of the OTDM-WDM system, we use an additional optical filter, referred to as the OTDM filter, and placed after OTDM demultiplexing but before photodetection as in Fig. 1. The OTDM filter used is a second order, super-Gaussian bandpass filter with bandwidth $B W_{\text {otdm }}$. The reasoning for the OTDM filter is that the OTDM demultiplexing process is basically a multiplication in the time domain. Thus, it is a convolution in the frequency domain. The resulting $10 \mathrm{~Gb} / \mathrm{s}$ demultiplexed channel spectrum is the convolution of the optically filtered $40 \mathrm{~Gb} / \mathrm{s}$ signal spectrum with the spectrum of the switching window. As a result, the demultiplexed channel becomes a wideband signal again with $10 \mathrm{GHz}$ harmonics. Putting the signal through a bandpass filter leads to a narrowband signal once again that would help minimize amplitude jitter.

\section{Performance Estimation Models}

For IM systems, the Gaussian approximated Q-factor is commonly used to estimate the BER because it gives a relatively good estimate, even though the actual noise distribution is not Gaussian [5]. It is estimated from the receiver current eye diagram as $Q_{I M}=\left|\mu_{1}-\mu_{0}\right| /\left(\sigma_{1}+\sigma_{0}\right)$ with BER $=(1 / 2)$ erfc $\left[Q_{I M} / \operatorname{sqrt}(2)\right]$ where $\mu_{1,0}$ and $\sigma_{1,0}$ are the mean and standard deviation of the marks/spaces rail [6]. Furthermore, a technique whereby the $\mathrm{Q}$ is conditioned to the received patterns of 3 bits can more accurately account for ISI [7] and is used here. When expressed in decibels, we use the common convention of $Q(\mathrm{~dB})=20 \log _{10}(Q)$.

\section{Results and Discussion}

We consider both a fully inline periodic DM system with $L_{i n l}=2 \mathrm{~km}$ and $L_{\text {pre }}=L_{\text {post }}=0 \mathrm{~km}$ and a lumped DM system with $L_{\text {inl }}=0 \mathrm{~km}$ and $L_{\text {pre }}=L_{\text {post }}=18 \mathrm{~km}$ The channel spacing $\Delta f$ is $97 \mathrm{GHz}$. We use a Q contour plot to optimize the optical filter bandwidths $B W_{w d m}$ and $B W_{o t d m}$. The electrical filter bandwidth $B W_{\text {ele }}$ is set at $B W_{\text {ele }}=\left(B W_{\text {otdm }}+10\right) \mathrm{GHz}$. It is interesting to note that without the OTDM filter the optimal performance is achieved for $B W_{w d m}=40 \mathrm{GHz}$, whereas with the inclusion of the OTDM filter, the optimal performance is achieved for $90 \mathrm{GHz}<B W_{w d m}<120 \mathrm{GHz}$ and $B W_{\text {otdm }} \sim 30 \mathrm{GHz}$. With the OTDM filter, the optimal WDM filter bandwidth is much higher. The wideband WDM filter allows a lot of noise and linear crosstalk into the signal while minimizing ISI. The task of filtering out the residual noise and crosstalk is then left to the narrowband OTDM filter. The OTDM filter bandwidth can be very narrow without inducing much ISI 


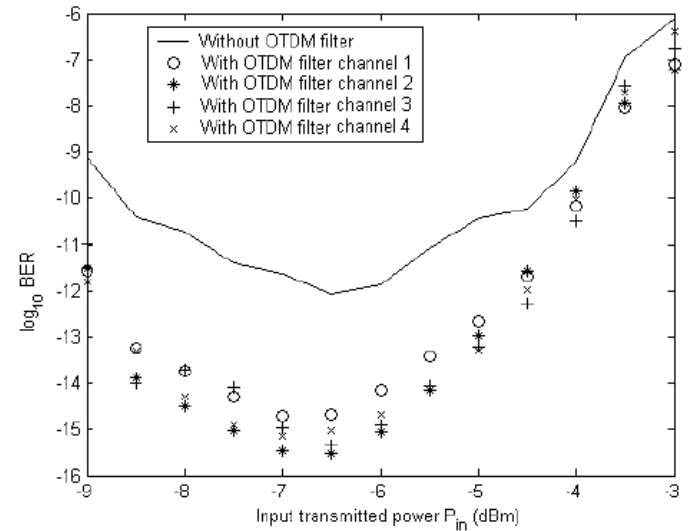

(a)

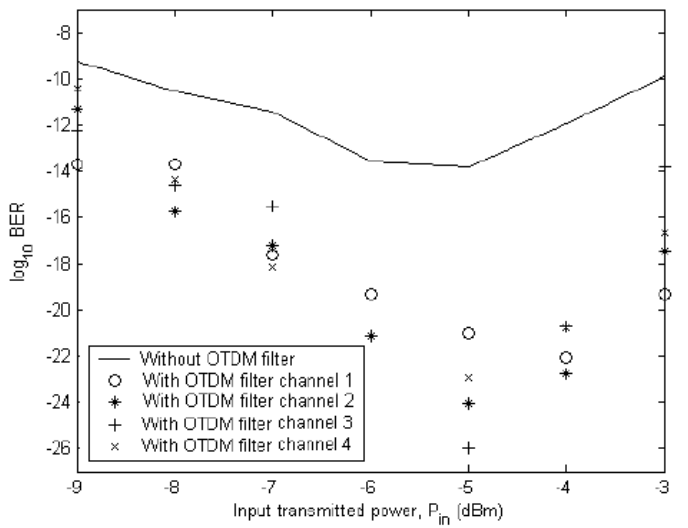

(b)

Fig. 2. BER vs. input transmitted power, $P_{\text {in }}$ for the cases with or without the OTDM filter $(\Delta f=$ $\left.97 \mathrm{GHz}, B W_{w d m}=110 \mathrm{GHz}, B W_{\text {otdm }}=15 \mathrm{GHz}\right)$; (a) Fully inline DM: $L_{\text {inl }}=2 \mathrm{~km}, L_{\text {pre }}=L_{\text {post }}=$ $0 \mathrm{~km}$ (b) Fully lumped DM: $L_{\text {inl }}=0 \mathrm{~km}, L_{\text {pre }}=$ $L_{\text {post }}=18 \mathrm{~km}$

because the time period between pulses for the $10 \mathrm{~Gb} / \mathrm{s}$ signal is much longer compared to the $40 \mathrm{~Gb} / \mathrm{s}$ signal. Thus, the post-OTDM-demultiplexed signal can tolerate ISI much better as the pulses can spread out further without encroaching into the space of neighboring pulses. The filter bandwidth values are set to $B W_{w d m}=110 \mathrm{GHz}$ and $B W_{\text {otdm }}=30 \mathrm{GHz}$ to obtain the results in Fig. 2. Comparing between the performances with and without the OTDM filter, there is significant improvement in the performance of the $10 \mathrm{~Gb} / \mathrm{s}$ demultiplexed channels with use of the very narrow OTDM filter that helps to reduce amplitude jitter without causing high ISI. This improvement occurs for both lumped and periodic DM with better overall performance achieved for lumped DM.

We test the distance limit of the OTDM-WDM system in Fig. 3 (a) by propagating the signal through $N$ spans of NZ-DSF using a fully inline DM scheme $\left(L_{\text {inl }}=2 \mathrm{~km}\right)$. The system shows good performance up to $N=38$ (total link length $=1976 \mathrm{~km}$ ) with the use of the OTDM filter providing a Q gain of $0.7-1.1 \mathrm{~dB}$. Notice that the optimum $P_{\text {in }}$ decreases as transmission distance increases indicating that nonlinear effects increases in significance compared to ASE noise for longer links.

Next, we reduce the channel spacing for higher spectral efficiency in Fig. 3 (b) using a hybrid DM scheme $\left(L_{i n l}=1 \mathrm{~km}, L_{\text {pre }}=L_{\text {post }}=9 \mathrm{~km}\right.$, total link length $=900 \mathrm{~km})$. The hybrid scheme shows improved performance over the inline scheme and the system performs well up to $\Delta f=77 \mathrm{GHz}$ which corresponds to a spectral efficiency of $0.52 \mathrm{bits} / \mathrm{s} / \mathrm{Hz}$. The optimal input power was $-5 \mathrm{dBm}$ for all cases. The optimal filter bandwidths remained the same as well. Notice that the Q gain due to the OTDM filter reduces as $\Delta f$ decreases, indicating that the narrowband filtering becomes less effective. This is because the closely spaced WDM channels induce more in-band linear crosstalk, which is difficult to filter out. 


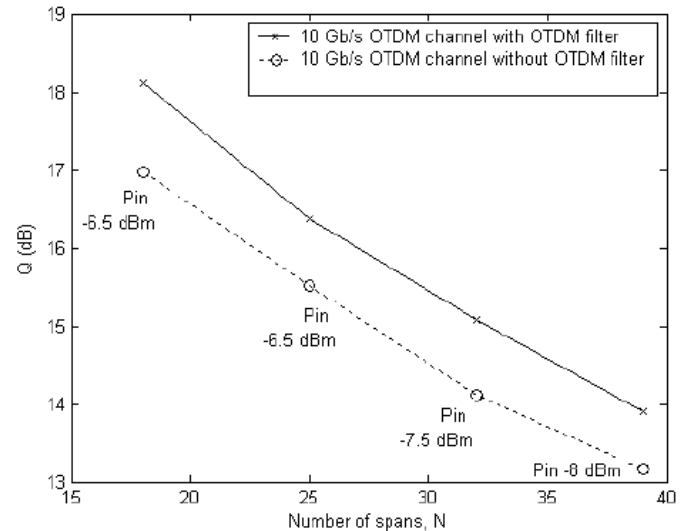

(a)

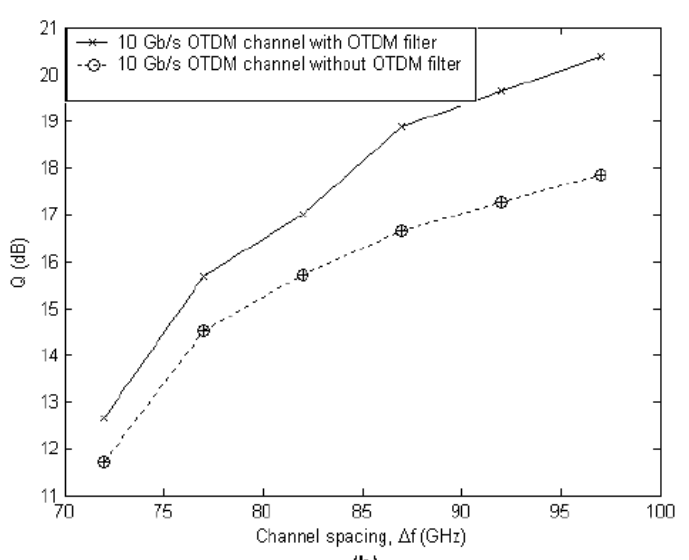

(b)

Fig. 3. (a) Optimal Q (dB) vs. number of spans, $N$ for receiver with or without the OTDM filter (optimum $P_{\text {in }}$ indicated and $L_{\text {inl }}=2 \mathrm{~km}, L_{\text {pre }}=L_{\text {post }}=$ $0 \mathrm{~km}, \Delta f=97 \mathrm{GHz})$. (b) Optimal $\mathrm{Q}(\mathrm{dB})$ vs. channel spacing, $\Delta f$ for receiver with or without the OTDM filter $\left(L_{\text {inl }}=1 \mathrm{~km}, L_{\text {pre }}=L_{\text {post }}=\right.$ $9 \mathrm{~km}$, total link length $=900 \mathrm{~km}$ )

\section{Conclusion}

An OTDM-WDM, IM system employing optical amplification and DM is analyzed, with and without the introduction of an optical narrowband bandpass filter placed right after OTDM demultiplexing, called the OTDM filter. The introduction of the OTDM filter improves the BER performance of the OTDM channels significantly by reducing amplitude jitter while possessing high tolerance towards ISI due to the wider time period between pulses. The OTDM-WDM link shows good performance up to $2000 \mathrm{~km}$ with the OTDM filter providing a $\mathrm{Q}$ gain of $0.7-1.1 \mathrm{~dB}$. The optimal input power reduces for longer distances due to increased significance of nonlinear effects. A $900 \mathrm{~km}$ link showed good performance for spectral efficiencies as low as $0.52 \mathrm{bits} / \mathrm{s} / \mathrm{Hz}$, though the Q gain provided by the OTDM filter reduces as channel spacing decreases.

\section{Acknowledgments}

The authors acknowledge the financial support given by the Malaysian Ministry of Science, Technology and Innovation through the IRPA Grant. 